\title{
Ocorrência e recrutamento larval de Teredinidae (Mollusca, Bivalvia) na região do Sistema Costeiro Cananéia-Iguape, São Paulo, Brasil
}

\author{
Daniela T. de Moraes ${ }^{1,2}$, Clemente Coelho Jr. ${ }^{3}$, Rafael C. G. da Cruz \& Sônia G. B. C. Lopes ${ }^{1}$
}

1. Departamento de Zoologia, Instituto de Biociências, Universidade de São Paulo, Caixa Postal 11.461, 05422-970 São Paulo, SP.

2. Endereço atual: Instituto Nacional de Metrologia, Qualidade e Tecnologia, INMETRO, Av. Nossa Senhora das Graças, 50, Xerém. 25250-020 Duque de Caxias, RJ. (dtmoraes@inmetro.gov.br)

3. Instituto de Ciências Biológicas, Universidade de Pernambuco, Rua Arnóbio Marques, 310, 50100-130 Santo Amaro, Recife, PE. (ccoelhojr@uol.com.br)

\begin{abstract}
The occurrence and larval recruitment of Teredinidae (Mollusca, Bivalvia) in Cananeia-Iguape Coastal System, São Paulo, Brazil. The occurrence and larval settlement of Teredinidae in Cananéia-Iguape Coastal System were observed for one year, by using laminated pinewood panels installed in three stations inside the mangrove forest (intertidal zone) and in one station, in the infralittoral. In the mangrove forest, the larval colonization occurred after four months in the most frequently flooded station and after eight months in the two less frequent flooded stations. In the infralittoral, the panels were highly infested after four months. Along the experiment, the number of individuals decreased, especially in the infralittoral, possibly due to competition between teredinids and pholadids for wood. Bankia fimbriatula was the most abundant teredinid, occurring in the infralittoral and in the mangrove area, followed by Bankia gouldi and Lyrodus floridanus, which occurred only in the infralittoral and Nausitora fusticula which was found only in the mangrove forest. In total, 704 individuals of Teredinidae were collected. Martesia striata, the single pholadid, was found in the infralittoral, and totaled 2,658 individuals.
\end{abstract}

KEYWORDS. Intertidal zone, infralittoral, mangrove forest, teredinids, pholadid.

RESUMO. A ocorrência e o recrutamento larval de Teredinidae na região do Sistema Costeiro Cananéia-Iguape foram acompanhados por doze meses utilizando-se coletores laminados de pinho, instalados em três estações dentro do bosque de mangue (mediolitoral) e em uma estação, localizada no infralitoral. No manguezal, a colonização por larvas de teredinídeos ocorreu após quatro meses de permanência dos coletores na estação mais frequentemente inundada pela maré e somente após oito meses nas duas estações com menor frequência de inundação. No infralitoral, após quatro meses, os coletores já estavam consideravelmente danificados. Ao longo do experimento, houve um decréscimo no número de teredinídeos, especialmente no infralitoral, ocasionado possivelmente pela competição por alimento e espaço entre teredinídeos e foladídeos. Foram encontrados 2.658 indivíduos, sendo 704 de Teredinidae e 1.954 de Pholadidae. Entre os teredinídeos, Bankia fimbriatula foi a espécie mais abundante tanto no infralitoral quanto no manguezal, seguida de Bankia gouldi e Lyrodus floridanus que ocorreram somente no infralitoral, e Nausitora fusticula encontrada somente no manguezal. Martesia striata, único foladídeo, ocorreu somente no infralitoral.

PALAVRAS-CHAVE. Zona intertidal, infralitoral, manguezal, teredinídeos, foladídeo.

Os Teredinidae são bivalves especializados na perfuração e digestão de madeira, ocorrendo, predominantemente, em ambiente marinho e de água salobra de regiões temperadas e tropicais, embora algumas espécies possam ocorrer em águas mais frias ou em salinidades próximas a zero (TURNER, 1966; BORGES et $a l ., 2014)$. São animais de grande importância econômica e ecológica, pois aceleram a degradação de estruturas de madeira, contribuindo para a reciclagem mais rápida dos macrodetritos vegetais (ROBERTSON, 1991).

No Brasil, as pesquisas com teredinídeos enfocaram o levantamento e a distribuição das espécies e a avaliação da infestação em substratos artificiais (MÜLLER \& LANA, 1986; Martins-Silva et al., 1988; Junqueira et al., 1989, 1991; JunQUeIRA \& SilVA, 1991; BARRETO et al., 1993; LopeS \& NARCHI, 1993) e em madeiras próprias do ambiente manguezal (LoPES \& NARCHI, 1997; LeONEL et al., 2002, 2006; FiLHo et al., 2008), bem como a resistência de certas espécies de madeira à perfuração desses animais (MiLANO \& Lopez, 1977/1978; Reis, 1995). Poucos têm sido os trabalhos enfocando outros aspectos, como a colonização da madeira pelas larvas a partir de um foco de infestação (VAROTTO \& BARRETO, 1998) e a resistência dos teredinídeos à exposição ao ar (OMENA et al., 1990) e à baixa salinidade
(BARRETO et al., 2000).

A ocorrência e a distribuição dos teredinídeos estão relacionadas com a disponibilidade de madeira, salinidade e temperatura. A temperatura e a salinidade têm influência maior sobre as larvas, pois estas requerem condições ótimas com relação a esses fatores (TURNER, 1966; RAYNER, 1979; Borges et al., 2014). Além disso, em regiões entremarés, a constante exposição dos troncos das árvores ao ar retarda o assentamento larval (LOPES \& NARCHI, 1997) e as flutuações de salinidade causadas por tempestades súbitas e intensas afetam a sobrevivência dos adultos (BARRETO et al., 2000; PaAlvast \& VAn der Velde, 2011).

O presente trabalho tem por objetivo verificar a ocorrência e o recrutamento larval das espécies de Teredinidae em ambiente intertidal (manguezal) e subtidal (estuário), comparando-os quanto à diversidade e distribuição das espécies.

\section{MATERIAIS E MÉTODOS}

O sistema Cananéia-Iguape ocupa uma área de quase $2.500 \mathrm{~km}^{2}$ e está situado no extremo sul do litoral paulista $\left(25^{\circ} 00^{\prime} \mathrm{S}\right.$ e $\left.47^{\circ} 30^{\prime} \mathrm{W}\right)$ (Fig. 1). 


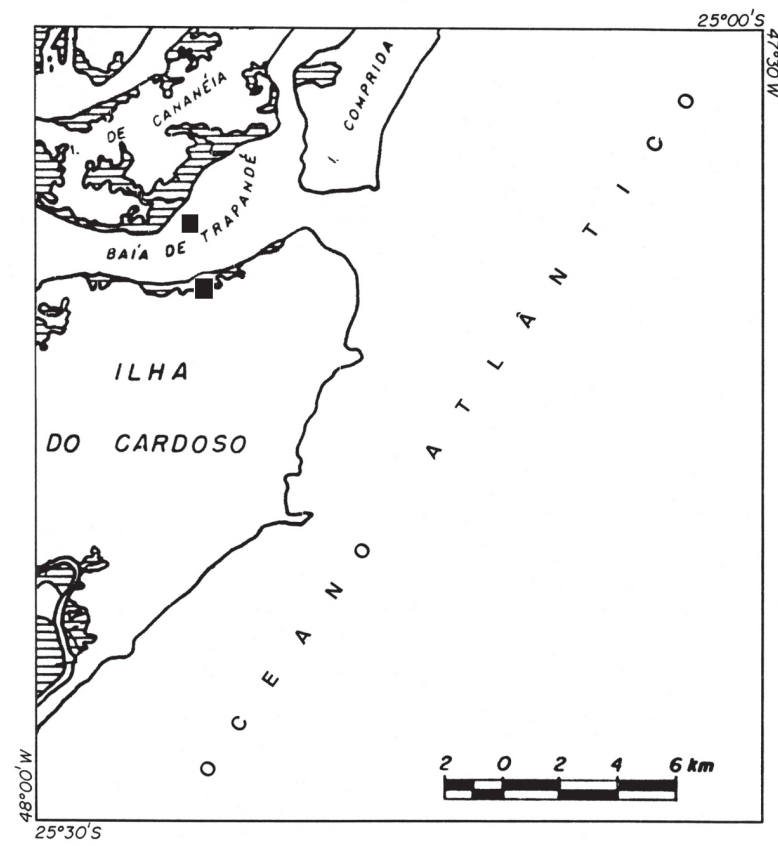

Fig. 1. Mapa da região do Sistema Costeiro Cananéia-Iguape, com o local onde foram instalados os coletores $($ - $)\left(25^{\circ} 04^{\prime} 58^{\prime}\right.$ 'S, $\left.47^{\circ} 57^{\prime} 42^{\prime \prime} \mathrm{W}\right)$ (adaptado de Herz, 1991).

Apresenta verões chuvosos e invernos secos, com valores pluviométricos máximos entre janeiro a março de 266,9 mm e mínimos em julho e agosto de 95,3 $\mathrm{mm}$. A temperatura média mensal mais alta $\left(25,2^{\circ} \mathrm{C}\right)$ ocorre em fevereiro e a mais baixa $\left(17,7^{\circ} \mathrm{C}\right.$ ) em julho (WAINER et al., 1996).

No manguezal da Ilha do Cardoso, onde foram estabelecidas as estações de coleta, há dominância de Rhizophora mangle L. em quase toda sua extensão, tanto em densidade de indivíduos quanto em desenvolvimento estrutural, exceto na faixa entre 40 e $60 \mathrm{~m}$ distante da baía de Trapandé, onde Avicennia schaueriana Stapf \& Leechman é codominante em área basal. O bosque de franja é bem desenvolvido, chegando a atingir $50,5 \mathrm{~m}^{2} /$ ha de área basal e altura média do dossel de $11,1 \mathrm{~m}$.
A salinidade média anual das águas das marés dentro do bosque de mangue foi de 29,4 \pm 2,0, extraída em poças de marés, enquanto que próxima ao estuário, na baía de Trapandé, foi de $28,3 \pm 1,0$. As amostras de água foram coletadas mensalmente e lidas com refratômetro óptico. A maré é do tipo mista, com predominância semidiurna e altura média de 0,81 m (Mesquita \& HARARI, 1983).

As estações 1, 2 e 3 localizam-se no manguezal da Ilha do Cardoso e distam 10, 40 e 80 metros da margem do bosque, respectivamente. Nessas estações, os coletores foram presos a cordas de náilon e amarrados às árvores, bem próximos ao substrato. A estação 4 localiza-se na Baía de Trapandé, no infralitoral. Os coletores ficaram frequentemente emersos durante a maré baixa nas estações 1,2 e 3 enquanto que os coletores na estação 4 ficaram sempre submersos a uma profundidade de cerca de $2 \mathrm{~m}$ (Fig. 2).

Em cada uma das estações foram instalados inicialmente 15 coletores laminados de pinho. A cada quatro meses, nas estações 1, 2 e 3, cinco coletores foram retirados para análise. Na estação 4, devido à grande infestação, dez coletores foram retirados depois dos primeiros quatro meses, sendo instalados mais 10. Após oito meses, 15 coletores foram retirados, sendo instalados mais 15 nesta estação.

O número total de coletores instalados nas quatro estações de coleta foi de 90 . O experimento teve início em abril de 2000 e término em abril de 2001.

Cada coletor é formado por 20 lâminas de pinho de $10,0 \times 10,0 \times 0,75 \mathrm{~cm}$, prensadas entre duas placas de acrílico de $10,0 \times 13,0 \times 0,3 \mathrm{~cm}$, presas por quatro parafusos de latão.

Realizou-se perfil microtopográfico perpendicular ao estuário, através do método de vasos comunicantes, com pontos equidistantes de um metro. Os perfis microtopográficos foram ajustados ao nível de redução, com dados referentes ao ano de 1996, obtidos no marégrafo instalado na Base João de Paiva Carvalho do Instituto Oceanográfico da Universidade de São Paulo em Cananéia,

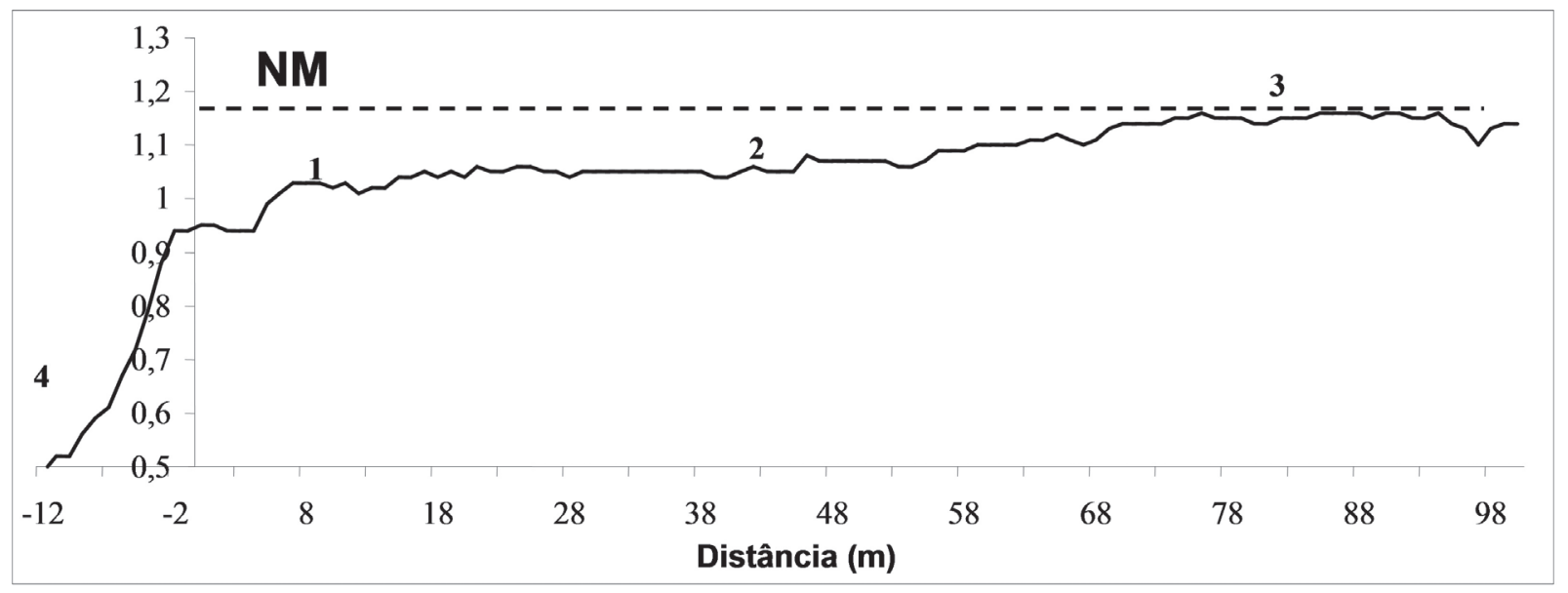

Fig. 2. Perfil topográfico do manguezal da Ilha do Cardoso, São Paulo, Brasil com os níveis médios das marés (NM, em metros) e a localização das estações de coleta $(1,2,3$ e 4$)$ ao longo do perfil. A distância do eixo X (em metros) refere-se à distância da margem do bosque de mangue. 
permitindo estimar a frequência de inundação ao longo do perfil.

A frequência de inundação (em \%) dentro do bosque do manguezal da Ilha do Cardoso está relacionada com a distância do estuário (em metros): (a) 0-20 m, 97,7\%; (b) 20-40 m, 93,3\%; (c) 40-60, 93,3\%; 60-80 m, 86,3\% e (e) 80-100 m, 86,3\%.

Depois de retirados de cada estação de coleta, os coletores foram levados ao laboratório, desmontados e os indivíduos removidos vivos foram fixados em formol $4 \%$ por 24 horas, medidos e transferidos para álcool $85 \%$ glicerinado (5:1).

O número de exemplares de cada espécie de teredinídeo por coletor foi calculado considerando os indivíduos retirados completos e as porções do corpo que continham as paletas. Os indivíduos foram identificados sob microscópio estereoscópico segundo TuRNER (1966). Teredinídeos sem as paletas, ou com paletas danificadas, ou mesmo muito pequenos, não puderam ser identificados ao nível específico.

A dominância de cada espécie foi estimada de acordo com Lopes \& NARchi (1997). A densidade de Teredinidae foi calculada dividindo-se a média de exemplares por coletor por $60 \mathrm{~cm}^{2}$, que representou a área da superfície do coletor exposta para colonização.

Dentre os indivíduos retirados completos, foram medidos 30 espécimes de Lyrodus floridanus (Bartsch, 1922), 62 de Bankia gouldi (Bartsch, 1908) e 224 de $B$. fimbriatula Moll \& Roch, 1931. Foram elaborados gráficos de frequência de indivíduos por classes de comprimento.

A fim de se comparar a ocorrência das espécies observadas utilizando coletores com aquelas existentes no manguezal, foram coletados troncos no interior do bosque de mangue, próximos aos locais de instalação dos coletores.

\section{RESULTADOS}

Nos coletores observou-se 2.658 animais perfuradores de madeira, sendo 704 teredinídeos (lotes
MZUSP 36899 a 36912, 40581 e 40582 ) e 1.954 foladídeos (lotes MZUSP 40579 e 40580).

As espécies de Teredinidae encontradas nos coletores foram, em ordem decrescente de abundância, B. fimbriatula, B. gouldi, L. floridanus e Nausitora fusticula (Jeffreys, 1860) (Tab. I), sendo que B. fimbriatula foi a única encontrada em coletores no infralitoral e em coletores e troncos coletados no interior do bosque de mangue. Martesia striata (Linnaeus, 1758), única espécie de foladídeo encontrada, representou aproximadamente $73 \%$ do total de bivalves perfuradores de madeira. Nos troncos coletados no interior do bosque de mangue ocorreram, em ordem decrescente de abundância, Neoteredo reynei (Bartsch, 1920), Nausitora fusticula e B. fimbriatula. Em troncos coletados próximos à margem, encontraram-se apenas espécimes de $B$. fimbriatula associados a Martesia striata. Não foi encontrado nenhum espécime de $N$. reynei nos coletores instalados no bosque de mangue durante o período de amostragem.

No manguezal, a colonização por larvas de teredinídeos na estação 1, a mais frequentemente inundada pela maré, ocorreu após quatro meses de permanência dos coletores no local. Nas estações 2 e 3, com menor frequência de inundação, a colonização ocorreu somente após oito meses. No infralitoral, depois de quatro meses de permanência no local, os coletores encontravam-se muito mais infestados do que no ambiente entremarés (Tab. II).

Ao longo do experimento verificou-se um decréscimo no número total de teredinídeos, especialmente no infralitoral. Após quatro meses, obteve-se um total de 383 indivíduos (95,3\% na estação 4). Após oito meses, o total foi de 289 indivíduos (78,9\% na estação 4) e, depois de 12 meses, somente 32 indivíduos foram coletados $(62,5 \%$ na estação 4) (Tab. I). Nas estações 1, 2 e 3, onde só ocorreram teredinídeos, a densidade populacional diminuiu no último período amostrado (Tab. II). Na estação 4, embora haja diminuição na densidade aos oito meses de experimento, aos 12 meses a densidade populacional aumentou, devido a um aumento no número de foladídeos.

Tab. I. Número de indivíduos das espécies de Teredinidae e Pholadidae nas estações de coleta no Sistema Costeiro Cananéia-Iguape, São Paulo, Brasil (M, mês de retirada dos coletores; $\mathrm{D} \%$, dominância em porcentagem; $\mathrm{N}$, número de indivíduos; $\mathrm{X}$, média de indivíduos por coletor).

\begin{tabular}{|c|c|c|c|c|c|c|c|c|c|c|c|}
\hline \multirow[b]{2}{*}{ Espécie } & \multirow[b]{2}{*}{ Est. } & \multicolumn{3}{|c|}{ M: agosto/2000 } & \multicolumn{3}{|c|}{ M: dezembro/2000 } & \multicolumn{3}{|c|}{ M: abril/2001 } & \multirow{2}{*}{$\begin{array}{c}\text { Total em } 12 \\
\text { meses }\end{array}$} \\
\hline & & $\mathrm{N}$ & $\mathrm{D} \%$ & $\mathrm{X}$ & $\mathrm{N}$ & $\mathrm{D} \%$ & $\mathrm{X}$ & $\mathrm{N}$ & $\mathrm{D} \%$ & $\mathrm{X}$ & \\
\hline \multirow{5}{*}{ B. fimbriatula } & 1 & 18 & 1,8 & 3,6 & 45 & 5,8 & 9,0 & 1 & 0,1 & 0,2 & 64 \\
\hline & 2 & & & & 1 & 0,1 & 0,2 & 1 & 0,1 & 0,2 & 2 \\
\hline & 3 & & & & & & & & & & \\
\hline & 4 & 302 & 30,1 & 30,2 & 99 & 12,8 & 6,6 & 18 & 2,0 & 1,2 & 419 \\
\hline & 1 & & & & 4 & 0,5 & 0,8 & 8 & 0,9 & 1,6 & 12 \\
\hline \multirow[t]{2}{*}{ N. fusticula } & 2 & & & & 4 & 0,5 & 0,8 & 1 & 0,1 & 0,2 & 5 \\
\hline & 3 & & & & 7 & 0,9 & 1,4 & 3 & 0,3 & 0,6 & 10 \\
\hline B. gouldi & 4 & 32 & 3,2 & 3,2 & 96 & 12,5 & 6,4 & & & & 128 \\
\hline L. floridanus & 4 & 31 & 3,1 & 3,1 & 33 & 4,3 & 2,2 & & & & 64 \\
\hline M. striata & 4 & 619 & 61,7 & 61,9 & 481 & 62,5 & 32,1 & 854 & 96,4 & 57 & 1954 \\
\hline Total & & 1002 & & & 770 & & & 886 & & & 2658 \\
\hline
\end{tabular}


Tab. II. Densidade de indivíduos por $\mathrm{cm}^{2}$ registrados nas quatro estações de coleta no Sistema Costeiro Cananéia-Iguape, São Paulo, Brasil durante os doze meses de amostragem (M, mês de retirada dos coletores).

\begin{tabular}{cccc}
\hline \multirow{2}{*}{ Estações } & M: agosto/2000 & M: dezembro/2000 & M: abril/2001 \\
\cline { 2 - 4 } & \multicolumn{3}{c}{ Densidade $\left(\mathrm{cm}^{2}\right)$} \\
\hline 1 & 0,06 & 0,16 & 0,03 \\
2 & 0 & 0,02 & 0,01 \\
3 & 0 & 0,02 & 0,01 \\
4 & 1,64 & 0,79 & 0,97 \\
\hline
\end{tabular}

Nos coletores da estação 4 a presença de teredinídeos é verificada no primeiro mês e, após três ou quatro meses, os coletores já se encontravam consideravelmente infestados (densidade $=0,75$ ind. $\left./ \mathrm{cm}^{2}\right)$.

No infralitoral, após quatro meses, B. gouldi (Fig. 3) teve maior incidência de indivíduos entre $4,1 \mathrm{e} 7,0 \mathrm{~cm}$, acompanhados de indivíduos de 1,1 a 3,0 cm. Esses dados sugerem que existiram duas épocas de recrutamento larval: uma logo no início do experimento, e outra mais recente, evidenciada pelos indivíduos menores. Para L. floridanus (Fig. 5) houve maior incidência de indivíduos pequenos, nas classes de 1,1 a $5,0 \mathrm{~cm}$, com maior frequência na classe de 2,1 a 3,0 cm, sugerindo uma infestação recente.

Após oito meses, os padrões de frequência para essas duas espécies foram semelhantes. Tanto B. gouldi (Fig. 4) quanto $L$. floridanus (Fig. 6) apresentaram incidência maior de indivíduos menores (entre 1,1 e 4,0 cm), sugerindo, novamente, uma infestação recente. Nenhum indivíduo de B. gouldi e L. floridanus foi coletado aos 12 meses de permanência dos coletores na estação 4 .

Bankia fimbriatula, a espécie mais abundante de teredinídeo, apresentou padrão de distribuição de indivíduos por classes de tamanho semelhante ao descrito para $L$. floridanus. Após quatro meses, B. fimbriatula (Fig. 7) teve maior incidência de indivíduos menores (entre 1,1 e 4,0 cm), sugerindo uma infestação recente. Após oito meses, B. fimbriatula (Fig. 8) também apresentou um maior número de indivíduos menores (entre 1,1 e 2,0 cm), sugerindo mais uma vez, uma infestação recente. Aos 12 meses obtiveram-se poucos indivíduos, não sendo possível constatar nenhum padrão de distribuição por classes de tamanho nessa amostra.

Nos coletores da estação 4 foi observada fauna abundante associada aos coletores (Anexo I).

\section{DISCUSSÃo}

A colonização da madeira por larvas de teredinídeos ocorre de forma mais rápida nas áreas do bosque de mangue mais frequentemente inundadas durante as preamares. $\mathrm{Na}$ estação 4, em que os coletores ficaram sempre submersos, a colonização por perfuradores foi ainda maior e mais rápida que na estação 1 . O tempo de submersão da madeira na água do mar é muito importante para a colonização mais rápida dos perfuradores. Quanto mais tempo a madeira estiver em contato com a água do mar, maior número de larvas pode entrar em contato com ela e iniciar a penetração.
Além do tempo de submersão, o tempo de exposição ao ar também pode ser um fator que interfira na sobrevivência das larvas e, consequentemente, afeta o assentamento larval. Sabe-se que até seis horas de exposição ao ar não ocorre mortalidade significativa em adultos (OMENA et al., 1990), mas não há muitos dados que avaliem o quanto as larvas são afetadas. OMENA et al. (1990) determinaram que a resistência de L. floridanus à emersão é diretamente proporcional ao comprimento do corpo. Estes autores sugeriram que espécies com paletas mais elaboradas, como as do gênero Bankia, poderiam ser menos suscetíveis à dessecação, por serem estas mais eficientes no fechamento da galeria. Por outro lado, LOPES $\&$ NARCHI (1993), ao estudarem a distribuição das espécies no Manguezal da Praia Dura, verificaram que, embora as espécies de Bankia e L. floridanus estivessem adaptadas a ambiente entremarés, necessitavam de imersões mais frequentes, pois eram encontradas nas áreas do bosque mais frequentemente inundadas pela maré, ao passo que Nausitora fusticula e Neoteredo reynei (que apresentam paletas menos elaboradas), eram registradas em áreas menos frequentemente inundadas, podendo permanecer intervalos maiores de tempo fora do contato com a água. Esses dados foram confirmados no presente trabalho. Bankia fimbriatula não foi encontrada na estação menos frequentemente inundada, enquanto que $N$. fusticula não foi registrada na estação mais frequentemente inundada pela maré. LeONEL et al. (2006) observaram redução do número de indivíduos de $B$. fimbriatula em segmentos do tronco de mangue mais frequentemente expostos ao ar.

A redução no número total de teredinídeos durante o período de amostragem pode estar relacionada à competição com os foladídeos pelo substrato, pelo menos no que se refere à estação 4, pois quando o número de foladídeos aumentou, reduziu-se o de teredinídeos drasticamente.

A salinidade é um fator que parece afetar, particularmente, o assentamento e a metamorfose das larvas de teredinídeos (RAYNER, 1979). BARRETO et al. (2000) verificaram que para as duas espécies mais comuns na Baía da Ilha Grande, RJ, a salinidade entre 11 e 13 era limitante para a sobrevivência dos indivíduos adultos. Scheltema \& Truitt (1954), Junqueira \& Silva (1991) e Lopes \& NARCHI (1993) observaram uma infestação decrescente com o aumento da distância da desembocadura dos rios no mar, destacando o papel da salinidade na distribuição dos teredinídeos nesse ambiente.

Segundo Scheltema \& TRUitT (1954) a maioria das espécies de teredinídeos é eurihalina. Em regiões estuarinas, L. floridanus e $B$. gouldi ficaram restritas às regiões com salinidades mais elevadas (MÜlLER \& LANA, 1986; LOPES $\&$ NARCHI, 1993). No presente trabalho estas duas espécies foram encontradas somente no infralitoral, onde não ficaram sujeitas às oscilações da maré, mas também a salinidade não apresentou muita variação em relação à obtida na margem do bosque de mangue, o que pode representar a importância do tempo de submersão na distribuição dessas 

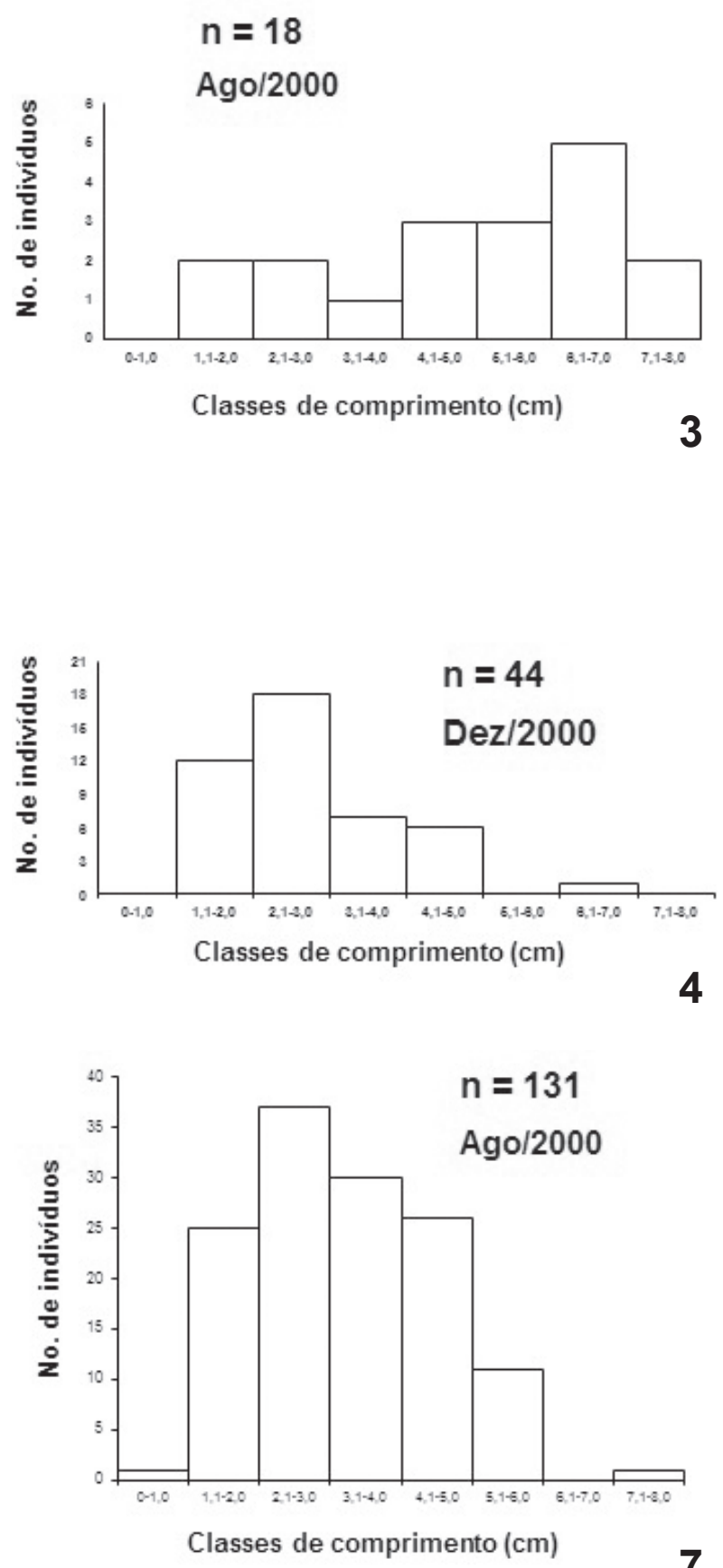



Classes de comprimento $(\mathrm{cm})$

5
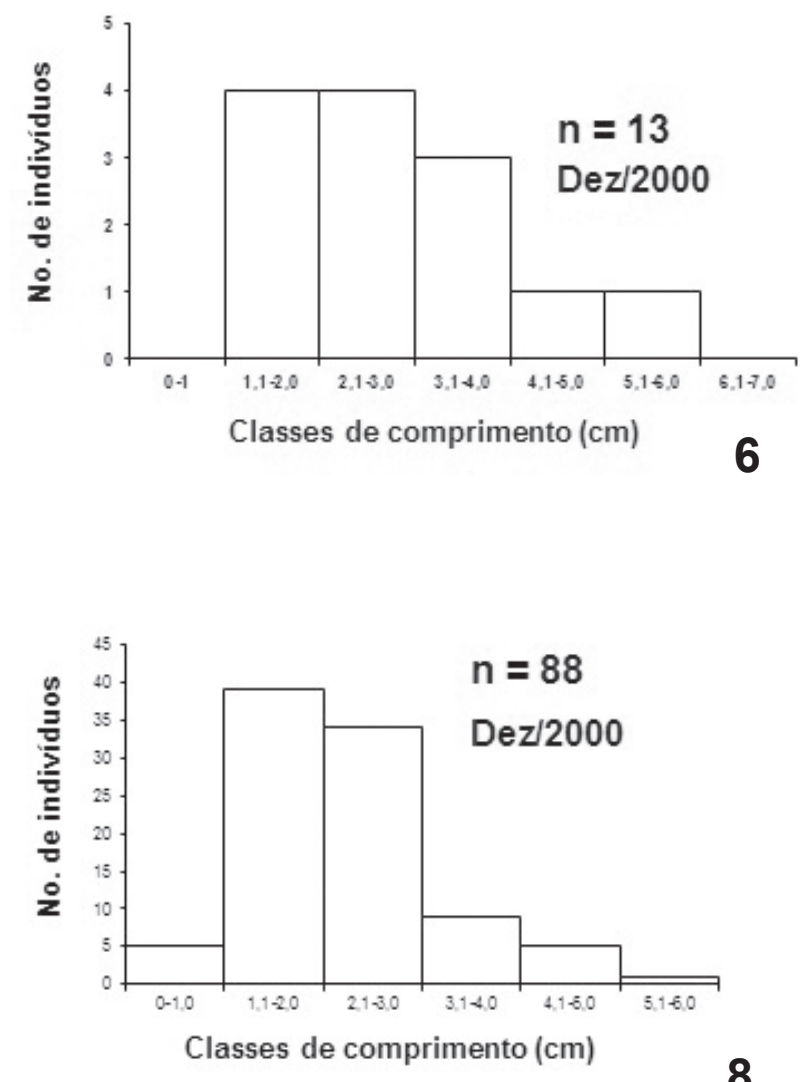

Figs 3-8. Número de indivíduos (n) por classes de comprimento (cm) após quatro e oito meses, respectivamente, de Bankia gouldi (3, 4), Lyrodus floridanus $(5,6)$, ambos coletados na estação 4, e Bankia fimbriatula $(7,8)$, coletados na estação 1, 2 e 4, Sistema Costeiro Cananéia-Iguape, São Paulo, Brasil.

espécies e não da salinidade como fator limitante. Teredo navalis, por exemplo, colonizou áreas do Mar Báltico onde a salinidade era mais alta, porém estabeleceu-se bem quando a salinidade decresceu, adaptando-se a essas condições (Borges et al., 2014).

O recrutamento larval de B. gouldi, L. floridanus e B. fimbriatula ocorreu a partir da instalação dos coletores, em eventos distintos de infestação, fato sugerido pela existência de classes de tamanho maiores e menores no mesmo intervalo de tempo. Para B. fimbriatula, Leonel et al. (2002) também verificaram maior recrutamento larval entre setembro e fevereiro, durante o período em que as salinidades estão mais altas naquela localidade. É provável que a presença de jovens de $B$. fimbriatula durante grande parte do período de amostragem indique que esta espécie pode ser encontrada o ano todo, como também 
o demonstrou Junqueira \& Silva (1991). A ausência de indivíduos de $B$. gouldi e $L$. floridanus e a presença de poucos espécimes de $B$. fimbriatula na última coleta impossibilitou uma melhor compreensão do período de recrutamento larval. Entretanto, sabe-se que, para algumas espécies de teredinídeos o crescimento larval é mais rápido em condições de salinidades acima de 20 (RAYNER, 1979) do que em salinidades mais baixas e, portanto em períodos chuvosos (neste caso, de dezembro a março) em que a salinidade decresce, provavelmente o recrutamento larval também seria menor.

É importante considerar que $B$. fimbriatula, $B$. gouldi e Nausitora fusticula possuem larvas planctotróficas e que a detecção de espécimes pequenos na população deve estar relacionada com um desenvolvimento larval prévio, ou seja, uma fase planctônica longa. Como os primeiros espécimes de $N$. fusticula foram encontrados em agosto, é provável que o período reprodutivo desta espécie ocorra no inverno, como sugerem LeONel et al. (2002). As espécies com larvas lecitotróficas apresentam período de vida planctônica mais curto que as planctotróficas e têm geralmente maiores chances de sucesso reprodutivo. Em função disso, geralmente são as mais abundantes (Turner, 1966; Borges et al., 2014). No entanto, o número de indivíduos de L. floridanus, única espécie com larvas lecitotróficas encontrada, foi pequeno quando comparado ao das espécies com larvas planctotróficas. Neste caso, é provável que, apesar do período de vida planctônica maior, as larvas planctotróficas estejam mais bem adaptadas às condições das regiões estuarinas, como sugerido por LOPES \& NARChi (1993) e LeONel et al. (2002, 2006). Estes últimos sugeriram que o número reduzido de T. barstchi coletado, uma espécie com larvas lecitotróficas, pode ser explicado pela competição com outros teredinídeos pela madeira e pela salinidade mais baixa.

A ausência de $N$. reynei nos coletores colocados no bosque de mangue não é um dado novo, pois também já havia sido anteriormente observada por MÜLLER \& LANA (1986) e ReIs (1995) em seus respectivos estudos. Esta espécie, embora não raro tenha sido coletada em troncos no bosque de mangue, não é frequentemente encontrada em coletores de pinho, nem em coletores de madeiras extraídas de espécies de manguezal (LOPES \& NARCHI, 1997). Esse fato pode estar relacionado à baixa incidência dessa espécie no bosque de mangue (LeONel et al., 2006).

A composição da fauna incrustante e vágil é dominada por crustáceos, seguida de moluscos e anelídeos, o que corrobora o observado por Aviz et al. (2009). JUNQUEIRA et al. (1989) referem-se à presença de incrustação biológica como um fator adicional que retardaria o assentamento das larvas de teredinídeos nos coletores, fato não avaliado no presente trabalho.

A colonização da madeira pelos teredinídeos está relacionada ao tempo de submersão da madeira e ao tempo de exposição ao ar na região do Sistema Costeiro CananéiaIguape. A colonização mais rápida da madeira deu-se no infralitoral onde os coletores permaneceram submersos durante todo o período de amostragem quando comparados aos coletores dispostos no bosque do manguezal, sujeitos à oscilação da maré.

Agradecimentos. Agradecemos à FAPESP pelo suporte financeiro e ao IO-USP pelo uso das instalações. Ao biólogo Norberto L. Hülle pelo auxílio nos trabalhos de campo. Aos Drs. Gustavo S. de Mello (MZUSP), Maria Teresa V. Berardo (Mackenzie-SP), João Miguel M. Nogueira (IB-USP) e André C. Morandini (IB-USP) pela identificação da fauna associada aos coletores.

\section{REFERÊNCIAS BIBLIOGRÁFICAS}

Aviz, D.; Mello, C. F. DE \& Silva, P. F. 2009. Macrofauna associada às galerias de Neoteredo reynei (Bartsch, 1920) (Mollusca: Bivalvia) em troncos de Rhizophora mangle Linnaeus durante o período menos chuvoso, em manguezal de São Caetano de Odivelas, Pará (costa norte do Brasil). Boletim do Museu Paraense Emílio Goeldi, Ciências Naturais 4 (1):47-55.

Barreto, C. C.; Silva, S. H. G. \& Lavrado, H. P. 1993. Distribution of teredinids along the Southeast coast of Brazil. Oebalia XIX (suppl.):155-162.

Barreto, C. C.; Junqueira, A. O. R. \& Silva, S. H. G. 2000. The effect of low salinity on Teredinids. Brazilian Archives of Biology and Technology 43(4):399-407.

Borges, L. M. S.; Merckelbach, L. M.; Sampaio, I. \& Cragg, S. M. 2014. Diversity, environmental requirements, and biogeography of bivalve wood-borers (Teredinidae) in European coastal waters. Frontiers in Zoology 11:13.

Filho, C. S.; Tagliaro, C. H. \& Beasley, C. R. 2008. Seasonal abundance of the shipworm Neoteredo reynei (Bivalvia,Teredinidae) in mangrove driftwood from a northern Brazilian beach. Iheringia, Série Zoologia 98(1):17-23.

Herz, R. 1991. Manguezais do Brasil. São Paulo, IOUSP-CIRM. 233p. Junqueira, A. O. R. \& Silva, S. H. G. 1991. Estudo experimental dos Teredinidae Rafinesque, 1815 (Mollusca: Bivalvia) do estuário da Lagoa da Tijuca, Rio de Janeiro, RJ, Brasil. Revista Brasileira de Biologia 51:113-126.

Junqueira, A. O. R.; Omena, E. P. \& Silva, S. H. G. 1991. A comparative study of the methods used to evaluate the activity of Teredinidae molluscs. Journal of Experimental Marine Biology and Ecology 150:107-115.

Junqueira, A. O. R.; Silva S. H. G. \& Silva, M. J. M. 1989. Avaliação da infestação e diversidade de Teredinidae (Mollusca, Bivalvia) ao longo da costa do Estado do Rio de Janeiro, Brasil. Memórias do Instituto Oswaldo Cruz 84(supl. IV):275-280.

Leonel, R. M. V.; Lopes, S. G. B. C. \& Aversari, M. 2002. Distribution of wood-boring bivalves in the Mamanguape River estuary, Paraíba, Brazil. Journal of Marine Biological Association of the United Kingdom 82:1039-1040.

Leonel, R. M. V; Lopes, S. G. B. C.; Moraes, D. T. de \& Aversari, M. 2006 The interference of methods in the collection of teredinids (Mollusca: Bivalvia) in mangrove habitats. Iheringia, Série Zoologia 96(1):25-30.

Lopes, S. G. B. C. \& NARChI, W. 1993. Levantamento e distribuição das espécies de Teredinidae (Mollusca, Bivalvia) no manguezal da Praia Dura, Ubatuba, São Paulo, Brasil. Boletim do Instituto Oceanográfico 41:29-38.

Lopes, S. G. B. C. \& NARCHI, W. 1997. Recrutamento larval e crescimento de Teredinidae (Mollusca-Bivalvia) em região entremarés de manguezais. Revista Brasileira de Oceanografia 45:77-88.

Martins-Silva, M. J.; Silva, S. H. G. \& Junqueira, A. O. R. 1988. Distribuição vertical de Teredinidae (Molusca, Bivalvia) em Portogallo, Angra dos Reis, R. J. Brasil. Revista Brasileira de Zoologia 5:155-165.

Mesquita, A. L. \& Harari, J. 1983. Tides and tide gauges of Cananéia and Ubatuba - Brasil (Lat $24^{\circ}$ ). Relatório interno do Instituto Oceanográfico 11:1-14.

Milano, S. \& Lopez, G. A. C. 1977/1978. Resistência natural de madeiras e xilófagos marinhos: resultados da primeira avaliação após três meses de instalação do ensaio. Preservação de Madeiras 8/9:31-42. 
MülleR, A. C. P. \& Lana, P. C. 1986. Teredinidae (Mollusca, Bivalvia) do litoral do Paraná. Nerítica 1:27-48.

Omena, E. P.; Junqueira, A. O. R. \& Silva, S. H. G. 1990. Resistência de Teredinidae Rafinesque, 1815 (Mollusca: Bivalvia) a diferentes períodos de exposição ao ar. Revista Brasileira de Biologia 50:701707.

Paalvast, P. \& van der Velde, G. 2011. Distribution, settlement and growth of first year individuals of the shipworm Teredo navalis $\mathrm{L}$ (Bivalvia: Teredinidae) in the Port of Rotterdam area, the Netherlands. International Biodeterioration and Biodegradation. 65:379-388.

RAYNER, S. M. 1979. Comparison of the salinity range tolerated by Teredinids (Mollusca: Teredinidae) under controlled conditions with that observed in an estuary in Papua New Guinea. Australian Journal of Marine and Freshwater Research 30(3):521-533.

ReIs, R. E. M. L. 1995. Moluscos bivalves perfuradores de madeira do estado do Pará, Brasil: caracterização taxonômica, distribuição e resistência de madeiras. Boletim do Museu Paraense Emilio Goeldi, Série Zoologia 11:125-203.
Robertson, A. I. 1991. Plant-animal interactions and the structure and function of mangrove forest ecosystems. Australian Journal of Ecology 16:433-443.

Scheltema, R. S. \& Truitt, R. V. 1954. Ecological factors related to the distribution of Bankia gouldi Bartsch in Chesapeake Bay. Chesapeake Biological Laboratory Publication 100:1-31.

Turner, R. D. 1966. A survey and illustrated catalogue of the Teredinidae (Mollusca: Bivalvia). Cambridge, Museum of Comparative Zoology. 265p.

VARotto, R. S. \& BARRETO, C. C. 1998. Colonization of artificial substrata by teredinid larvae released from a previously infested focus at Ilha Grande Bay, RJ. Brazilian Archives of Biology and Technology 41:391-400.

Wainer, I. E. K. C.; Colombo, P. M. \& Miguel, A. J. 1996. Boletim de monitoramento climatológico para as Bases Norte e "Dr. João de Paiva Carvalho" do Instituto Oceanográfico da Universidade de São Paulo. Relatório Técnico do Instituto Oceanográfico 38:1-13.

Anexo I.Fauna associada aos coletores instalados no infralitoral (estação 4), no Sistema Costeiro Cananéia-Iguape, São Paulo, Brasil.

Crustáceos

Panopeus occidentalis de Saussure, 1857

Panopeus austrobesus Williams, 1983

Panopeus americanus de Saussure, 1857

Hexapanopeus schimitti Rathbun, 1930

Caprella equilibra Say, 1818

Caprella scaura Templeton, 1836

Podocerus brasiliensis (Dana, 1853)

Ericthonius brasiliensis (Dana, 1853)

Stenothoe valida Dana, 1855

Photis longicaudata (Bate \& Westwood, 1868)

cracas

Anelídeos

Neanthes succinea (Frey \& Leuckart, 1847)

serpulídeo

Moluscos

Sphenia antillensis Dall \& Simpson, 1901

Mexilhões

ostras

Platelmintos

turbelários 